\title{
Atypical lipomatous tumour of the round ligament - report of a rare case with review of literature
}

\author{
R. Ray ${ }^{1}$, D. Biswas 2 , S. Mitra ${ }^{3}$ \\ 1 Department of General Surgery, College of Medicine and JNM Hospital, Kalyani, India \\ 2 Department of Obstetrics \& Gynecology, ESI-PGIMSR (Manicktala), Kolkata, India \\ 3 Department of Pathology, College of Medicine \& JNM Hospital, Kalyani, India
}

Keywords: Atypical; lipomatous; tumour; round ligament; well differentiated; liposarcoma

\section{Case report}

28 year old Asian female from Eastern India, presented with a swelling in the left groin of two months duration. A globular swelling of $10 \mathrm{~cm} \times 7 \mathrm{~cm}$ with soft to firm consistency was found in the left inguinal region. The swelling was irreducible and cough impulse test was equivocal.

It was misdiagnosed as irreducible inguinal hernia and elective hernioplasty was planned. Preoperative investigations were unremarkable. On exposure of the inguinal canal, a lobulated greyish white nodular mass attached to the round ligament was found. And there was no hernia sac. The mass along with the round ligament was excised up to the deep ring. The histopathology revealed it as an atypical lipomatous tumour. Patient is currently without any evidence of recurrence at three years follow up.

\section{Literature review and discussion}

Atypical lipomatous tumours (ALT) are low grade liposarcomas with propensity to local recurrence, but potential to dedifferentiate to higher grades [1]. In practice the term "well differentiated liposarcoma" is used for neoplasms occurring in locations where wide resection margins cannot be achieved easily e.g. abdomen, retroperitoneum. In contrast the term "atypical lipomatous tumor" is used for subcutaneous, subfascial, intramuscular tumours of extremities or trunk where wide margins can be achieved.

ALTs usually arise in deep soft tissue of the thigh retroperitoneum, mediastinum, paratesticular area and in the subcutaneous tissue. Liposarcomas of spermatic cord present as paratesticular masses with a reported incidence of three to seven percent [2]. These tumours are morphologically either adipocytic, sclerosing, inflammatory or spindle cell subtype.

Correspondence: R. Ray

E-mail: rubikray22@outlook.com

DOI: http://10.4038/sljs.v34i4.8322
Benign mesenchymal tumours originating from the round ligament are usually located intra abdominally [3]. However, a search of literature did not reveal any reported case of ALT arising in the inguinal region.

The peak incidence is between the fifth and seventh decade, although cases have been reported in younger age groups. These tumours usually present as deep seated, slow growing, painless masses that can attain large sizes. As in our case ALTs occurring in the inguinal or paratesticular region can be mistaken for irreducible or incarcerated inguinal hernias [4].

In a suspected case, ultrasound of the groin is usually the first imaging investigation. Typically these appear hyperechoic with well-defined margins. Features suggesting malignant variant with dedifferentiation include age ( $>60$ years ), size $(>10 \mathrm{~cm})$, male gender, presence of thick septa $(>2 \mathrm{~mm}$ ), presence of nodular, globular and non-adipose masses like areas with decreased percentage of fat [5]. These features are more readily appreciated on CT or MRI. Septal enhancement on contrast MRI may differentiate between well differentiated liposarcomas and simple lipomas. In a study including 126 patients, MRI was found to have a $100 \%$ sensitivity, $83 \%$ specificity, $84 \%$ accuracy and $38 \%$ positive predictive value in identifying liposarcomas. MRI was also $100 \%$ specific in diagnosis of simple lipoma [6]. Thus we recommend MRI in all cases of suspected lipomatous groin masses.

For ALT of the spermatic cord, en bloc removal of the tumour with negative margins by inguinal orchidectomy is recommended. No further therapeutic advantage is obtained by inguinal or retroperitoneal lymph node dissection owing to the low metastatic potential of ALTs.

There is no role of adjuvant radiotherapy or chemotherapy at present. However, radiotherapy may be recommended in case of positive margins, recurrences or aggressive histology [7]. Prognosis of ALTs is good owing to low risk of metastasis. However, these tumours can dedifferentiate to higher grade tumours. Dedifferentiation is defined as, abrupt transition in the primary tumour or recurrence to a nonlipogenic sarcoma. It is seen in up to $10 \%$ of cases. The dedifferentiated variety has less favourable prognosis with a local recurrence rate of 
$41 \%$, metastasis in $17 \%$ and 5 years disease specific mortality of $28 \%$ [8].

In our case although it was initially misdiagnosed as an irreducible hernia, intraoperative finding of a tumour with atypical appearance and heterogenous composition, prompted an en bloc resection with the round ligament up to the deep ring as a precautionary step. This turned out to be the correct decision postoperatively.

Therefore, in case of an unusual lipomatous groin mass it is important to maintain a high index of suspicion for a possible liposarcoma. In such a situation care must be taken to resect the mass completely to negative margins. Failure to do that may result in a recurrence of the tumour.

Dedifferentiation can only be determined from histopathology and postoperatively. It may prompt the surgeon to consider further resectional procedure or radiotherapy and thereby significantly increases morbidity for the patient.

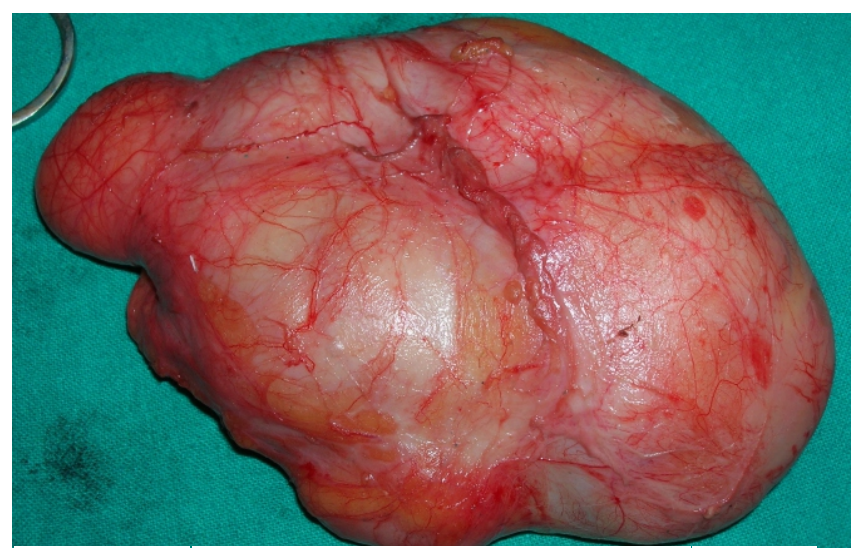

Figure 1. Specimen - Gross morphology - showing lobulated soft to firm mass

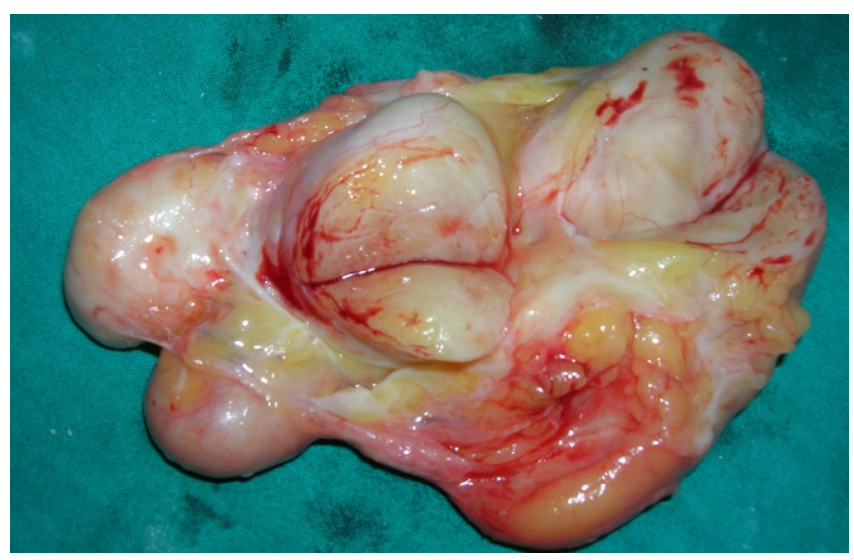

Figure 2. Specimen - Cut section - showing yellowish areas of fat interspersed with nodules whitish fibrous tissue and other soft tissue elements

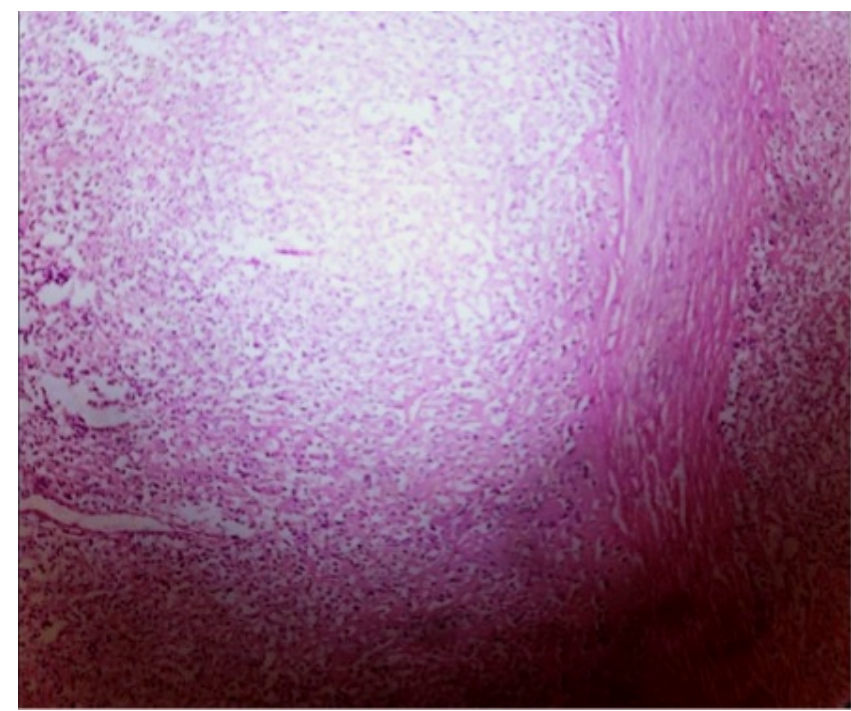

Microsections show lipoma like cells in lobular pattern separated by thick fibrous septa. (100x magnification, H\&E stain)

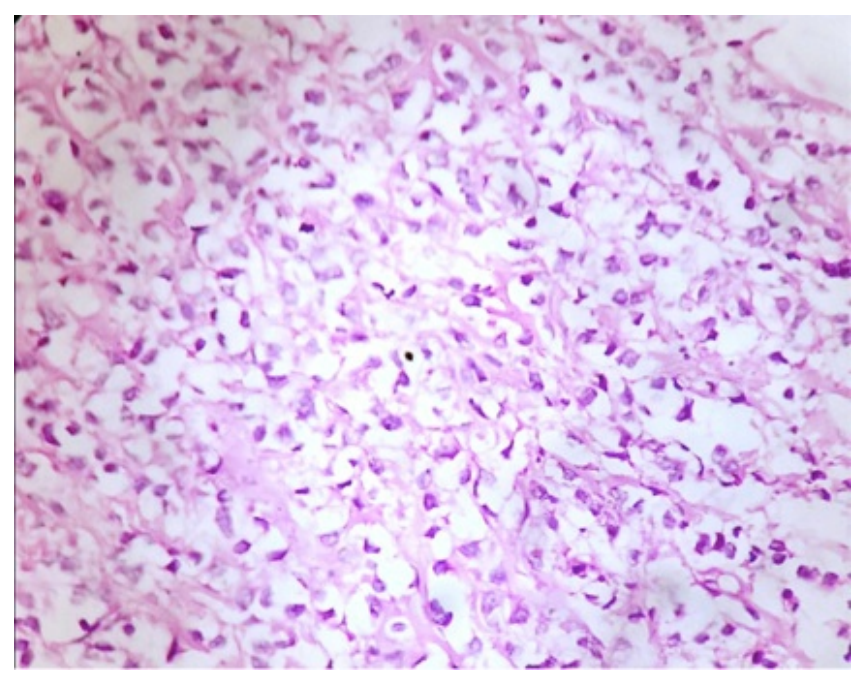

Microsections show malignant cells in sheets, they are round cells having vacuolated cytoplasm with irregular hyperchromatic nucleus. (400x magnification, H\&E stain)

\section{References}

1. Andreas F. Mavrogenis, M D ; Jan Lesensky, M D ; Carlo Romagnoli, MD; Marco Alberghini, MD; G. Douglas Letson, MD; PietroRuggieri, MD, PhD: Atypical Lipomatous Tumors/Well-differentiated Liposarcomas: Clinical Outcome of 67 Patients. Orthopaedics December 2011 - Volume 34 Issue 12: e893-e898

2. Kakuta Y, Kawamura N, Fukuhara S, Imadu T, Hara T, Yamaguchi S, Adachi S. Two cases of liposarcoma of spermatic cord. Hinyokika Kiyo. 2008 Feb;54(2):147-50.

3. Nuovo MA, Nuovo GJ, Smith D, Lewis SH. Benign mesenchymoma of the round ligament. A report of two cases with immunohistochemistry. Am J ClinPathol. 1990 Mar;93(3):421-4. https://doi.org/10.1093/ajcp/93.3.421

4. Joo - Lee Koh, Hung Lin, Ying -Huei Lee, Ching-Nan Lin. Paratesticular Liposarcoma Mimicking an Incarcerated Inguinal Hernia: Case Report and Literature Review. JTUA Sep. 2005; 16 (3):124-126

5. Kransdorf MJ, Bancroft LW, Peterson JJ, Murphey MD, Foster 
WC, Temple HT. Imaging of fatty tumors: distinction of lipoma and well-differentiated liposarcoma. Radiology. $2002 \mathrm{Jul}$; 224(1):99-104

https://doi.org/10.1148/radiol.2241011113

6. Cree M. Gaskin1 and Clyde A . Helms. Lipomas, Lipoma Variants, and Well-Differentiated Liposarcomas (Atypical Lipomas): Results of MRI Evaluations of 126 Consecutive Fatty Masses. Am J Roentgenol. March 2004;82(3):733-739

7. HaiderAlyousef, Elsawi M. Osman, and Mohamed A. Gomha,
"ParatesticularLiposarcoma: A Case Report and Review of the Literature," Case Reports in Urology, vol. 2013, Article ID 806289, 3 pages, 2013. doi:10.1155/2013/806289 https://doi.org/10.1155/2013/806289

8. Henricks W H, Chu Y C, Goldblum J R, Weiss S W . Dedifferentiated liposarcoma: a clinicopathological analysis of 155 cases with a proposal for an expanded definition of dedifferentiation. Am J SurgPathol 1997;21:271-81. https://doi.org/10.1097/00000478-199703000-00002

\section{Key Points:}

- Atypical lipomatous tumours are well differentiated liposarcomas and not a benign entity, with propensity for local recurrence and potential to dedifferentiate to higher grades over time.

- Majority of such tumours in groin occur in males as paratesticular masses but can also occur in females in inguinal region as seen in this rare case

- MRI is the most useful investigation to differentiate these tumours from simple lipomas which are benign

- Surgical resection to negative margins is adequate treatment, radiotherapy is required for positive margins or unfavourable histology to prevent local recurrence or metastasis 